\title{
Intravital Microscopy as a Tool to Study Intracellular Membrane Trafficking During Invasion and Metastasis
}

Panomwat Amornphimoltham ${ }^{1}$, Kamil Rechache ${ }^{1}$, Kantima Leelahavanichkul ${ }^{2}$, Alfredo Molinolo ${ }^{2}$, Vyomesh Patel $^{2}$, Silvio J. Gutkind ${ }^{2}$, and Roberto Weigert ${ }^{1}$ *

${ }^{1}$ Intracellular Membrane Trafficking Unit and ${ }^{2}$ Molecular Carcinogenesis Unit, Oral and Pharyngeal Cancer Branch National Institute of Dental and Craniofacial Research, National Institutes of Health 30 Convent Dr. 303A, Bethesda, MD 20892-4340

To study the role of membrane trafficking in invasion and metastasis in head and neck cancer, we utilized a human squamous carcinoma cell (HSCC) line, OSCC3 that shows a metastatic behavior when orthotopically implanted in the tongue of immuno-compromised mice [1]. In this model, OSCC3 cells formed a tumor 7-10 days after the injection into the tongue and invaded $80-100 \%$ of the cervical lymph nodes within 2-3 weeks. One of the major issues to be addressed is the mechanism of invasion and migration. To this aim we have developed an experimental system to perform repeated long-term imaging of the implanted tumor in the same animal. OSSC 3 cells have been modified to stably express various fluorescently tagged molecules such as pVenus or GFPH2B and then injected into the tongue of immuno-compromised mice. Three to four days after injection, each animal was anesthetized, the tongue retracted and placed in a custom-made positioning device, and an area on the edge of the tumor was imaged. The animals were anesthetized and imaged on a daily basis using collagen fibers on the surface of the tongue as reference points to locate the same areas of the tumor. Preliminary results suggest that single cells detached from the tumor mass, suggesting that the invasion process is mediated by single cells rather than collective migration. Furthermore, we were able to image single cells in the edge of the tumor highlighting their protrusive phenotype. Based on data coming from an extensive microarray analysis, we have identified a subset of genes implicated in membrane trafficking that are differentially expressed in oral cancer metastasis. Among them, we focused on RAB25, a small GTPases essential for the recycling of membrane proteins to the plasma membrane that is down-regulated in metastatic cells as determined by both RT-PCR and Western blot analysis [2,3]. Strikingly, the ectopical expression of RAB25 in the metastatic cells significantly reduced cell invasion and metastatic both in in vitro and in vivo models, whereas shRNA-mediated ablation of RAB25 in non-metastatic cells resulted in an invasive behavior. Very importantly, we have also demonstrated that lost of Rab25 is common in advance head and neck cancer patients. Our result, both in vitro and in vivo demonstrated the ability of Rab25 to prevent cell invasion and metastasis is associated with Rab25 c-terminal region (last 40 amino acids). Our results strongly suggest that Rab25 is a molecule that involve in actin cytoskeleton arrangement engages in interactions that prevent cells to migrate.

\section{References:}

[1] Patel V. et al., Cancer Research, 2011 (in press)

[2] Nam KT, et al., J. Clin Invest., 2010 120(3):840-9

[3] Caswell PT, Dev Cell 2007 13(4):496-510 